\title{
Super-lattice, rhombus, square, and hexagonal standing waves in magnetically driven ferrofluid surface
}

\author{
Hyun-Jae Pi, So-yeon Park, Jysoo Lee, and Kyoung J. Lee $\$$ \\ National Creative Research Initiative Center for Neuro-dynamics \\ and Department of Physics, Korea University, Seoul 136-701, Korea
}

(Dec 2, 1999)

Standing wave patterns that arise on the surface of ferrofluids by (single frequency) parametric forcing with an ac magnetic field are investigated experimentally. Depending on the frequency and amplitude of the forcing, the system exhibits various patterns including a superlattice and subharmonic rhombuses as well as conventional harmonic hexagons and subharmonic squares. The superlattice arises in a bicritical situation where harmonic and subharmonic modes collide. The rhombic pattern arises due to the non-monotonic dispersion relation of a ferrofluid.

47.20.k; 47.54.+r; 05.70.Fh

The phenomena of self-organization in spatially extended nonequilibrium systems have attracted much attention lately in the scientific community world-wide [1]. One of the recent scientific issues in this regard is to investigate the system in which a finite number of unstable (spatial and temporal) modes interact each other, while the past studies have mostly focussed on the limiting cases of either pattern forming systems where a single mode is excited or systems that are highly turbulent with many modes. Although the mode-coupling interactions have been a long standing issue in various fields ranging from material science to plasma physics, their significance in nonequilibrium pattern formation is only begun to be understood.

In a recent experiment of parametrically driven surface waves, Edwards and Fauve used a two-frequency excitation scheme and were able to generate a quasi-crystalline standing wave pattern with 12 -fold symmetry [2]. In subsequent experiments on a similar experimental setup, Kudrolli et al. observed two different types of superlattice pattern ("type-I" and "type-II") in addition to the 12 -fold symmetric quasicrystalline pattern [3]. More recently, Arbell and Fineberg report yet another kinds of superlattice pattern ("SSS" and "2MS") [4]. All these unusual patterns are obtained in twofrequency forced Faraday experiments in which two externally excited modes interact (i. e. near bicriticality). Very recently, however, Wagner et al. have demonstrated that such bicritical condition can be achieved even by a single frequency forcing for a viscoelastic liquid [5] or for a normal Newtonian fluid in an extreme driving condition (very shallow filling depth and large shaking elevations) [6]. New types of superlattice pattern are revealed in those systems. The existence of these unusual patterns all together suggests a large class of previously unanticipated spatio-temporal patterns with multiply interacting modes.
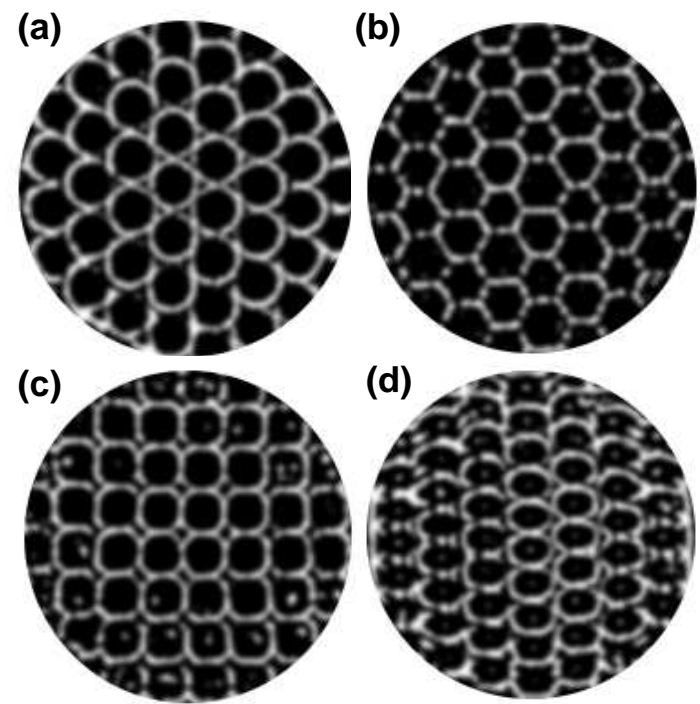

FIG. 1. Regular patterns observed on the surface of a driven ferrofluid in a cylindrical container: (a) harmonic hexagon ( $f=6.0$ $\mathrm{Hz}$ ), (b) subharmonic superlattice $(f=6.7 \mathrm{~Hz})$, (c) subharmonic square $(f=11.0 \mathrm{~Hz})$, and (d) subharmonic rhombus $(f=20.0$ $\mathrm{Hz})$. The total magnetic field is $H(t)=H_{0}+\Delta H \sin (2 \pi f t)$, where $H_{0} / H_{c}$ and $\Delta H / H_{c}$ are fixed at 0.94 and 0.22 , respectively. The diameter of the viewing area is $60 \mathrm{~mm}$.

In the present paper, we take a step in this direction by experimental study on a vertically oscillating ferrofluid that is driven by a sinusoidal magnetic field. There are two reasons for choosing the new system over previously investigated ones. First of all, the driven ferrofluid system can be easily brought near to a bicritical situation with a single frequency forcing - the harmonic mode as well as subharmonic mode can be generated by an ac magnetic field with a small driving frequency $(<30 \mathrm{~Hz})$ and a small magnetic field $(<100$ gauss $)$ [7]. This contrasts with the case of mechanically driven Newtonian fluids in which either a complex two-frequency forcing scheme or an extreme driving condition is necessary to achieve a bicritical situation [ [8]. The other reason is that ferrofluid exposed to a magnetic field can exhibit a non-monotonic dispersion relation [9]. Due to the nonmonotonicity the neural stability curve for the excitation of standing waves can have multiple minima [10,11], thus, several spatial modes can be excited simultaneously.

As expected, the driven ferrofluid system is quite rich to reveal a variety of standing wave patterns as shown in Fig. 1. Two unusual patterns, (1) superlattice with a mixed-mode oscillation and (2) subharmonic rhombic pattern, as well as 
usual subharmonic square and harmonic hexagonal patterns are observed. The observed superlattice is similar but different from any of the previously observed ones in its shape and temporal evolution. The rhombic pattern is the first example of its kind ever demonstrated in experiments on nonequilibrium pattern formation.

The ferrofluid is a colloidal suspension of magnetic powder stabilized by screened electrostatic repulsion [9]. We use a commercially available ferrofluid (EMG901, Ferrofluidics) [density $\rho=1.53 \mathrm{gm} / \mathrm{ml}$, surface tension $\sigma=29.5 \mathrm{~g} / \mathrm{s}^{2}$, initial magnetic susceptibility $\chi=3.00$, magnetic saturation $M_{s}=600$ gauss, dynamic viscosity $\eta=10 \mathrm{cp}$, yielding a critical field of the static Rosensweig-instability $H_{c}=79.73$ gauss]. A cylindrical Teflon container containing the ferrofluid (depth $=5 \mathrm{~mm}$, diameter $=85 \mathrm{~mm}$ ) is placed in the center of a pair of Helmholtz-coils (Cenco instruments) with a inner (outer) diameter of $100 \mathrm{~mm}(300 \mathrm{~mm})$. The magnetic field is monitored by means of a hall probe (F. W. Bell Inc., Model 4048), and the spatial variation of the field strength from the center to the outer rim is within 3\%. An ac signal is generated from a home-built synthesizer-board and amplified by a linear amplifier driving the total magnetic field of $H(t)=H_{0}+\Delta H \sin (2 \pi f t) . H_{0}$ is the static field, $\Delta H$ is the amplitude of ac component, and $f$ is the driving frequency. $H_{0}$ is fixed at $0.94 H_{c}$ for all cases, while $\Delta H$ and $f$ are used as a control parameter.

The fluid surface is illuminated by two arrays of concentric LED rings (diameter $100 \mathrm{~mm}$ and $120 \mathrm{~mm}$, respectively) located $230 \mathrm{~mm}$ above the surface. The flat surfaces either above or below the level of the surrounding fluid appear white, while the non-flat surfaces that scatter the light away from the camera appear black. The patterns are imaged using a high speed $256 \times 256$ pixel CCD camera (Dalsa, CA-D6-0256W) located $370 \mathrm{~mm}$ above the surface with a fast frame grabber (Matrox, Meteor-II). Maximum frame acquisition rate is 955 frame/s, enabling us to photograph distinct phases of moving pattern.

The phase diagram shown in Fig. 2 2 summarizes our results. Upon increasing sequence of $\Delta H$, the flat surface can be unstable to various crystalline states depending on the value of driving frequency $f$. Squares and rhombuses occur over a wide range of parameter space, whereas hexagons and superlattice form only in a narrow range at low driving frequency. Spatio-temporal chaotic state (STC) gradually forms with increasing number of defects as $\Delta H$ increases. Essentially, the same phase diagram is obtained with a square container.

We first focus on the sequence of bifurcations that occur with increasing value of $f$ for a fixed value of $\Delta H / H_{c}=$ 0.22 . The flat fluid surface becomes unstable first to a harmonic mode for a small $f$. Since the up-down symmetry is broken for a harmonic mode, quadratic interaction is the most significant nonlinearity [1], producing hexagonal standing wave pattern that is synchronous to the ac driving [Fig. 11(a) and Fig. 3 (a)]. With a gradual increase in $f$, the wave number $\left(k_{H}\right)$ increases slightly [see Fig. 3(e)] but the hexagonal symmetry does not change until $f=6.7 \mathrm{~Hz}$ at which the sys-

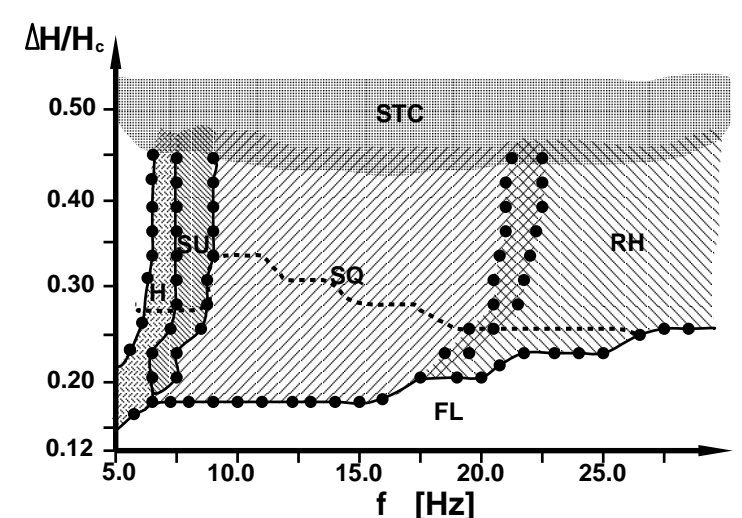

FIG. 2. Phase diagram of standing wave patterns revealed in a magnetically driven ferrofluid with $H_{0}=0.94 H_{c}$ : flat surface (FL), hexagon $(\mathrm{H})$, superlattice (SU), square (SQ), and rhombus $(\mathrm{RH})$. The thin shaded area between SQ and RH is bistable (i. e. SQ and RH coexist). Spatio-temporal chaotic state (STC) is generally observed at large $\Delta H$. Crossing the dashed line toward the STC state, the crystalline states start to acquire defects.
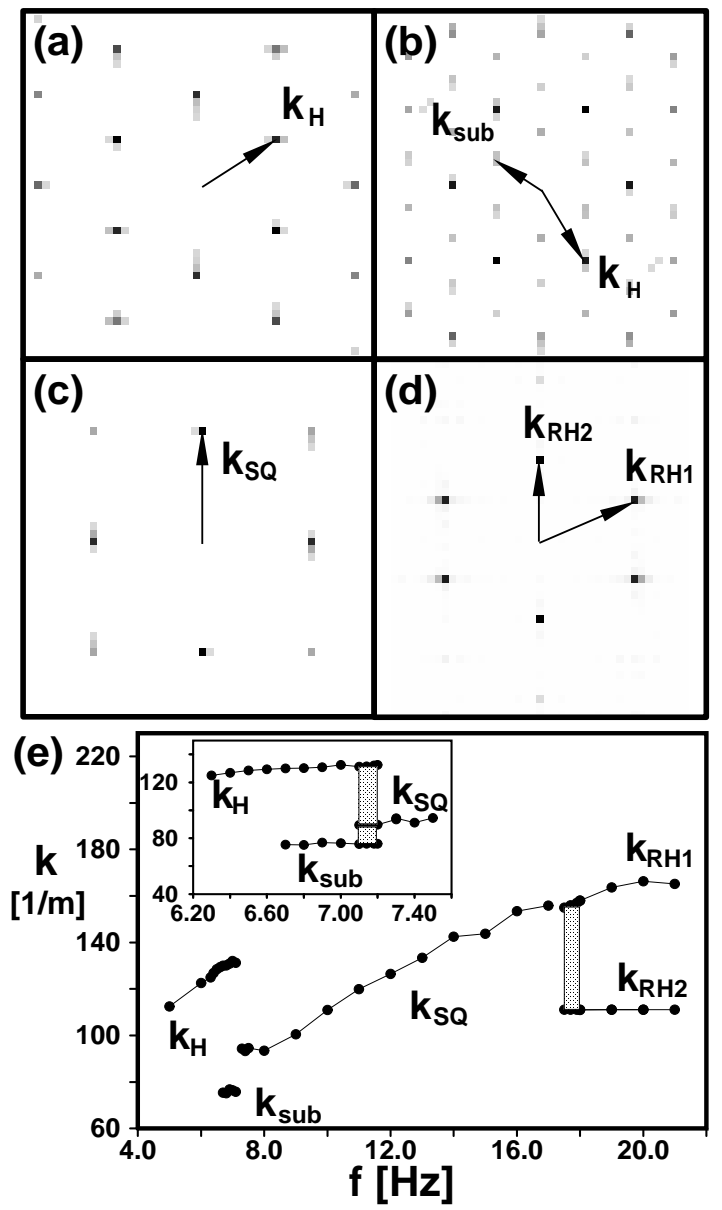

FIG. 3. Fourier transforms of the regular patterns shown in Fig. 1: (a) hexagon, (b) superlattice, (c) square, and (d) rhombus. The magnitude of wavevectors associated with different patterns vs $f$ are shown in (e). The insets shows the details of the transition among hexagon, superlattice, and square. 
(a)
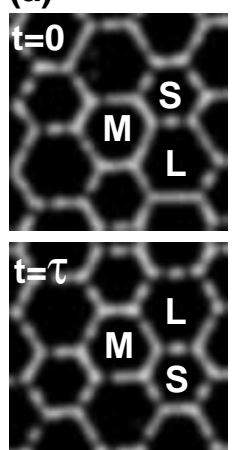

(b)
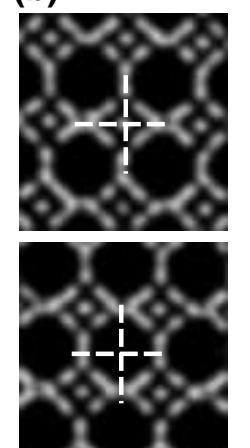

(c)

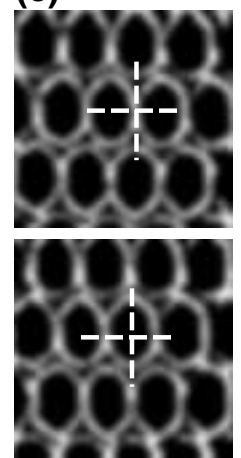

FIG. 4. Time evolution of subharmonic patterns during one driving cycle: (a) superlattice, (b) square, and (c) rhombus. The patterns after one driving period $(\tau)$ are shown in the bottom row. The dashed cross lines are placed to indicated the same location.

tem enters into the bicritical region. At the onset of bicriticality, 6 subharmonic peaks $\left(k_{\text {sub }}=k_{H} / \sqrt{3}\right)$ suddenly appear forming a superlattice pattern together with the primary 6 harmonic peaks [see Fig. 1.(b) and Fig. 3(b)]. The transition from hexagon to superlattice is very sharp and appears to be first order, but the exact nature of the transition could not be resolved within the accuracy of our current experimental setup.

The observed superlattice pattern is similar but different from the ones previously observed by others [3-6]. In particular, the snapshot of superlattice [Fig. 4 (a)] taken at $t=0$ is basically tiled by three different cells differing in sizes and shapes. During one driving cycle the cell " $\mathrm{M}$ " returns to the same "M", while "S" and "L" swap each other. Upon completing the subsequent driving cycle, "M" returns to the same, but "S" and "L" swap again. In other words, the superlattice in Fig. 1(b) has a mixed mode oscillation. This is in marked contrast with the purely subharmonic hexagonal superlattices observed by Wagner et al. that alternates between two complementary hexagonal lattices composed with cells of same type [5], and also with the "Type-II superlattice" observed by $\mathrm{Ku}$ drolli et al. that undergoes a complex stripe modulation during the course of oscillation [3].

Since the superlattice forms in a narrow region between harmonic hexagon and subharmonic square, it is natural to believe that the superlattice forms by an interplay between the two different modes. Since both harmonic and subharmonic modes are present, dominant nonlinearity of inter-mode interaction is quadratic generating new subharmonic modes. In addition, translational invariance requires that the newly generated wavevectors are linear combinations of $k_{H}$ and $k_{S Q}$. They would in turn interact with the dominant harmonic hexagonal basis, and only those which satisfy the (spatial and temporal) resonance conditions would survive [12]. Two out of four subharmonic square modes $k_{S Q}$ and four out of new subharmonic modes satisfy the resonance conditions, and form a hexagonal sublattice with magnitude $k_{\text {sub }}=k_{H} / \sqrt{3}$, thus contributing to the superlattice. The present mechanism for the generation of the superlattice is very similar to that of [6].
As $f$ is increased further, the superlattice undergoes a hysteretic transition to a subharmonic square lattice; upon increasing (decreasing) sequence of $f$, the superlattice (square) transforms to the square (superlattice) at $f=7.10 \mathrm{~Hz}(f=$ $7.20 \mathrm{~Hz}$ ). At the onset of transition to square lattice, $k_{S Q}$ is approximately $k_{H} / \sqrt{2}$. In the bistable regime, mixed states of square domains and superlattice domains are often seen. The observed square patterns are subharmonic just like the ones that are popularly seen in conventional Faraday experiments: the pattern alternates between two dual square lattices at every driving period [see Fig. 4 (b)]. The $k_{S Q}$ increases significantly as $f$ increases.

Upon increasing $f$ further, the square lattice undergoes a hysteretic transition to a stable rhombic pattern with a well defined 2-fold symmetry [see Fig. B(d) and (e)]. At the transition, the $k_{S Q}$ branch continues to $k_{R H 1}$ (primary rhombic basis vector) and a new basis vector $k_{R H 2}$ that is the difference between two neighboring $k_{R H 1} \mathrm{~s}$ is spontaneously created. The oblique angle between $k_{R H 1}$ and $k_{R H 2}$ characterizes a particular rhombic pattern. For the example shown in Fig. 3(d), $k_{R H 2} / k_{R H 1}=0.696$ and the angle between the two neighboring basis wavevectors is $69.7^{0}$. The oblique angle changes but slightly as the driving frequency changes (less than $2 \%$ for the frequency range $17.5-21.0 \mathrm{~Hz}$ ). The system is bistable for the range $(17.5 \mathrm{~Hz}<f<18.0 \mathrm{~Hz})$. Except for the bistable region, the rhombic lattice is globally stable. Figure $A$ (c) clearly demonstrate that the observed rhombic pattern is temporally subharmonic just like the square pattern shown in Fig. 4(b). As far as we know, the observed rhombic pattern is the first laboratory demonstration of its kind ever revealed [13].

At this point, we note that a recent theoretical study has shown that parametrically driven surface waves on ferrofluids can be unstable to multiple spatial modes simultaneously [10]. Here, the existence of multiple unstable modes originates from the non-monotonic dispersion relation of ferrofluids. The study has shown that in one-dimensional geometry one can realize a situation that different domains with different wavevectors coexist. Such phenomenon is also verified recently in an experiment conducted in a quasi one-dimensional annular geometry [11]. The existence of rhombic pattern in our system, however, suggests that in two-dimensional space the (two) unstable modes rather form a deformed crystalline structure (i. e. rhombic lattice), instead of domains with different wavevectors. In our experiments, coexisting domains with different wavevectors are only observed in bistable regions. In order to check the feasibility of this hypothesis, we have extended the earlier theoretical study by Raitt and Riecke [10] to two-dimensional space and have found a parameter regime in which well defined rhombic patterns are globally stable [14]. Recently, Lifshitz and Petrich have also demonstrated the existence of a stable rhombic pattern in a generic model system containing two unstable spatial modes [15].

In the following, the transition to spatio-temporal chaotic state (STC, see Fig 28) from crystalline states is qualitatively described. As $\Delta H$ increases for a fixed value of $f$, the crys- 
(a)

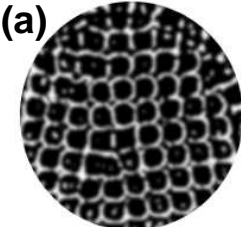

(b)
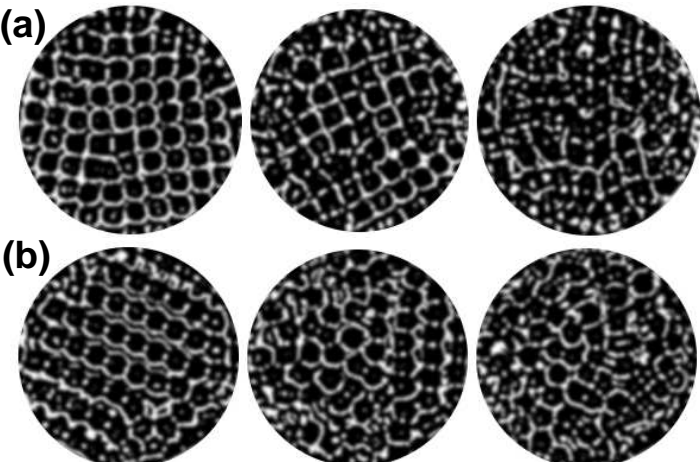

FIG. 5. Transitions to disordered patterns from (a) square and (b) rhombus, as $\Delta H$ increases. The frequency $f$ is fixed at $15.0 \mathrm{~Hz}$ for (a) and $21.0 \mathrm{~Hz}$ for (b). $\Delta H / H_{c}$ is $0.28,0.39,0.45$ (from left to right) for (a), and $0.28,0.37,0.45$ for (b).

talline patterns gradually become disordered. Figure 5 shows two such sequences, one for the case of square and the other for the case of rhombic pattern. Dislocations and defects start to form in a square lattice near $\Delta H / H_{c}=0.28$ [Fig. F(a), left image]. As $\Delta H$ increases, the number of defects increases (middle image) and eventually the whole lattice becomes very loose as in a typical melting process. The last image in Fig. 5(a) shows a state very near to STC, showing a very little trace of square lattice. A similar melting process is also observed for the case of rhombic lattice [Fig. 5(b)]. The gradual deformation toward the state of STC is not limited to the square and rhombus but occurs in the whole range of $f$ that we have explored. This observation is consistent with the earlier Faraday experiments conducted with Newtonian fluids [16].

In conclusion, superlattice and rhombus, as well as square and hexagonal standing waves, are observed on the surface of ferrofluids driven parametrically with an ac magnetic field. It is argued that the superlattice pattern is formed by the resonance of newly generated subharmonic modes with the harmonic hexagonal mode already present. Due to the nonmonotonic dispersion relation of the ferrofluids, multiple spatial modes can become unstable simultaneously with a single frequency forcing. As a consequence, stable rhombic patterns are possible and observed in the experiment. Full understanding of the observed patterns and their mutual boundaries, however, pose quite a challenge.

We are indebted to W. G. Choe for many helpful discussions. This work is supported by Creative Research Initiatives of the Korean Ministry of Science and Technology.

+ To whom correspondence should be addressed (Email: kyoung@nld.korea.ac.kr).

[1] See, e.g., M. Cross and P. Hohenberg, Rev. Mod. Phys. 65, 851 (1993).
[2] W. S. Edwards and S. Fauve, J. Fluid Mech. 278, 123 (1994); Phys. Rev. E 47, R788 (1993).

[3] A. Kudrolli, B. Pier, and J. P. Gollub, Physica D 123, 99 (1998).

[4] H. Arbell and J. Fineberg, Phys. Rev. Lett. 81, 4384 (1998).

[5] C. Wagner, H. W. Müller, and K. Knorr, Phys. Rev. Lett. 83, 308 (1999).

[6] C. Wagner, H. W. Müller, and K. Knorr, "Crossover from a square to a hexagonal pattern in Faraday surface waves", pattsol/9911001 (1999).

[7] J. -C. Bacri, U. d'Ortona, and D. Salin, Phys. Rev. Lett. 67, 50 (1991); T. Mahr and I. Rehberg, Europhys. Lett. 43, 23 (1998).

[8] H. Müller, H. Wittmer, C. Wagner, J. Albers, K. Knorr, Phys. Rev. Lett. 78, 2357 (1997).

[9] R. Rosenweig, Ferrohydrodynamics, Cambridge University Press, Cambridge (1985).

[10] D. Raitt and H. Riecke, Phys. Rev. E 55, 5448 (1997).

[11] T. Mahr and I. Rehberg, Phys. Rev. Lett. 81, 89 (1998).

[12] M. Silber and M. R. E. Proctor, Phys. Rev. Lett. 81, 2450 (1998); M. Silber and A. C. Skeldon, Phys. Rev. E 59, 5446 (1999).

[13] Experimental evidence of stable rhombic pattern was discussed earlier in a chemical reaction-diffusion system by Q. Ouyang et. al [Chaos 3, 707 (1993)]. However, their observation is markedly different from ours: their rhombic lattice has a broad spectrum of angles with a mean value of $60^{\circ}$, whereas our rhombic lattice is essentially tiled by a single rhombus with an oblique angle far different from $60^{\circ}$.

[14] J. Lee and K. Lee, to be published.

[15] R. Lifshitz and D. M. Petrich, Phys. Rev. Lett. 79, 1261 (1997).

[16] N. Tufillaro, R. Ramshankar and J. Gollub, Phys. Rev. Lett. 62, 422 (1989); A. Kudrolli and J. P. Gollub, Physica D 97, 133 (1996). 